\title{
A Simulation Framework to Automatically Analyze the Communication-Computation Overlap in Scientific Applications
}

\author{
Vladimir Subotic, Jose Carlos Sancho \\ Barcelona Supercomputing Center \\ Spain \\ vladimir.subotic@bsc.es, jsancho@bsc.es
}

\author{
Jesus Labarta, Mateo Valero \\ Barcelona Supercomputing Center \\ Universitat Politècnica de Catalunya \\ Spain \\ jesus.labarta@bsc.es,mateo.valero@bsc.es
}

\begin{abstract}
Overlapping communication and computation has been devised as an attractive technique to alleviate the huge application's network requirements at large scale. Overlapping will allow to fully or partially hide the long communication delays suffered when transferring messages through the network. This will relax the application's network requirements, and hence allow to deploy more cost-effective network designs. However, today's scientific applications make little use of overlapping. In addition, there is no support to analyze how overlap could impact the performance of real scientific applications. In this paper we address this issue by presenting a simulation framework to automatically analyze the benefits of communication-computation overlap. The simulation framework consists of a binary translation tool (Valgrind), a distributed machine simulator (Dimemas), and a visualization tool (Paraver). Valgrind instruments the legacy MPI application and generates the execution traces, then Dimemas uses the obtained traces and reconstructs the application's time-behavior on a configurable parallel platform, and finally Paraver visualizes the obtained time-behaviors. Our simulation methodology brings two new features into the study of overlap: 1) automatic simulation of the overlapped execution - as there is no need for code restructuring in applications; and 2) visualization of simulated time behaviors, that further allows useful comparisons of the non-overlapped and the overlapped executions.
\end{abstract}

Keywords: MPI; Communication-Computation Overlap;

\section{INTRODUCTION}

In a parallel computer, communication delays might substantially decrease the application performance, specially at large scale. The traditional practice to alleviate this problem is to use a high performance interconnects that provide system-wide high bandwidth and low latency. An example of these networks is the Mellanox InfiniBand Quad data rate (QDR) switches [20] that provides $8 \mathrm{Gbp} / \mathrm{s}$ of effective unidirectional bandwidth per link. Network implementers can aggregate multiple of those links to significantly increase the bandwidth provided to applications; 4, 8, or 12 links can be aggregated together to provide $32 \mathrm{~Gb} / \mathrm{s}, 64 \mathrm{~Gb} / \mathrm{s}$, $96 \mathrm{~Gb} / \mathrm{s}$ bandwidth respectively. However, as a parallel machine deploys higher bandwidth, the cost of its network becomes a significant part of the total cost of the whole system [10]. This current trend in network design does not seem to be sustainable forcing the research community to explore new techniques. One of these techniques is to find ways to profit more from the already existing cycles in the network. One solution to optimize network usage is to overlap communication delays with useful computation of the application. This way, the application's computation can hide communications delays, and thus the network requirements can be substantially relaxed.

The state-of-the-art networks already provide excellent support for overlapping communication and computation. The current networks, such as Myrinet [4], introduced the OS-bypass technology in which the network interface can perform communication operations without interrupting the main processor. Hence, the processor and the network can simultaneously compute and transfer data, allowing full overlap of computation and communication.

However, it still remains unclear how much real scientific applications can benefit from overlap. Today's large scientific applications are mostly based on MPI and follow the Bulk-Synchronous programming model (BSP). These applications try to maximize the network bandwidth by aggregating all the communications into one message. Consequently, the application's computations and communications are explicitly separated - applications compute and then communicate with minimal overlap between these two phases. On the other hand, code optimizations that aim to increase communication-computation overlap are cumbersome and demand a lot of programming time. Furthermore, it is hard to anticipate how much these optimizations can improve real applications, so the programmer cannot know in advance whether the code restructuring is worth the effort. Therefore, our goal in this work is to offer to the community a fast and precise simulation framework that estimates how much a real scientific MPI application can benefit from increased overlap.

In this paper we present a simulation framework that quantifies the potential benefits of automatic communication-computation overlap in scientific MPI applications. Up to our knowledge, this is the first work that uses a simulation methodology to study overlap. The 
simulated overlapping technique works at the MPI level, by automatically capturing all MPI messages and trying to overlap those messages with useful computation of the application. The overlapping technique consists of the following mechanisms: message chunking, advancing sends, double buffering, and post-postponing receptions. These mechanisms are further described below.

Our framework is automatic and easy-to-use approach to obtain a rich simulated output that can significantly increase the overall understanding of communication-computation overlap. The simulation framework allows us to get predictions quicker, and furthermore evaluate the impact of different network environments. The main advantage of this approach is that it automatically predicts the benefits of overlap in scientific MPI applications, without the need to know or understand the application's source code. The second advantage is that our simulation framework can visualize the simulation's output allowing us to qualitatively inspect differences between the non-overlapped and overlapped executions. Using this feature, for example, an implementer can easily identify bottlenecks in the overlapping technique and try to fix them.

The rest of this paper is organized as follows. In Section II we briefly describe the technique to exploit the overlap for MPI applications. Next in Section III we describe our simulation framework in order to analyze the communicationcomputation overlap in scientific applications. Section IV and Section V describe the experimental setup and the obtained results. Section VI summarizes recent work in the field of overlap. And finally, conclusions are given in Section VII.

\section{COMmUNiCATION-COMPUTATION OVERLAP AT THE MPI LEVEL}

Overlapping communication and computation at the MPI level consists of overlapping MPI transfers with the computation in which the data elements of these MPI transfers are produced and consumed. This can be achieved using the following four techniques.

- Message chunking: Each original MPI message is partitioned into independent chunks consisting of one or more data elements.

- Advancing sends: Each chunk is sent as soon as it is produced.

- Double buffering: Two different buffers are used to differentiate the chunks being consumed at the current iteration and the incoming chunks for consuming at the next iteration.

- Post-postponing receptions: Each chunk is waited at the moment when it is really needed for consumption.

Figure 1 shows the traditional case of non-overlapped MPI communications. Here, the process $A$ has to wait until the MPI message that consist of four data elements is fully produced during the iteration $i$. Then the MPI message is sent to process $B$ for computing in the next iteration $i+1$. Therefore, there is no overlap of the communication of the MPI message with any of the computation phases in the iterations $i$ and $i+1$, and thus, both processes, sender and receiver, are suffering the corresponding communication delays.

Conversely, Figure 2 shows the case of using the four mentioned techniques to overlap MPI transfers between the two processes. Here, the application can overlap the transfer delays by 1) splitting the message into smaller chunks in this case, each message is composed of four chunks, 2) at the sender side, sending each chunk as soon as it is produced; and 3) at the receiver side, postponing the reception of chunks until the corresponding data elements are really needed for consumption. Therefore the application can overlap the communication of a chunk with the computation that produces succeeding chunks at the sender side and with the computation that consumes preceding chunks at the receivers side. For example the communication delays of the chunk $p_{1}$ can be overlapped with the computational time to produce the following chunks $p_{2}$ and $p_{3}\left(T p_{2}+T p_{3}\right)$ and also with the computational time to consume the chunk $c_{0}\left(T c_{0}\right)$. In general, the communication of a chunk $i$ can be overlapped with the following computation times:

$$
\sum_{i+1}^{n-1} T p_{j}+\sum_{0}^{i-1} T c_{j}, 0 \leq i \leq n-1
$$

where $T p_{j}$ and $T c_{j}$ are the production and consumption time intervals to process the chunk $j$, and $n$ is the total number of chunks in a MPI message.

Additionally, the double buffering technique is used to prevent overwriting of the communicated data at the receiver side. As illustrated in Figure 2, the chunk $p_{0}$ might arrive to process $B$ during the iteration $i$, instead of at the next iteration $i+1$. Therefore, it can conflict with the previous value $p_{0}$ that is already there. The double buffering technique solves this problem by storing the message for the iteration $i+1$ in a different buffer from the values used in the current iteration $i$.

It is important to note that the equation above describes the ideal case where the computation time available to overlap the transfers is the higher one possible for all chunks. However, an application can use a different production/consumption pattern that might not be as favorable for overlap as the pattern above, and thus the total amount of computational time available for overlap may be drastically reduced. For example, if an application consumes first the last produced chunk, there is no computational time available to overlap this particular chunk. Even worse, if an application produces and consumes all chunks at the same time, there is no computational time available to overlap any of the chunks. The diversity of production/consumption patterns and their influence on the overlapping potential will 


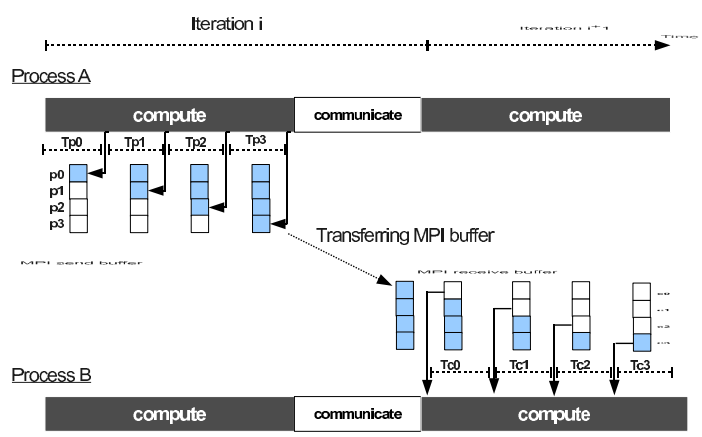

Figure 1. Case of non-overlapping communication and computation.

\section{Simulation FramewORK}

The simulation framework is based on the integration of three well-known tools: the binary translation tool Valgrind [21], the network simulator Dimemas [14], and the visualization tool Paraver [22] as it is shown in Figure 3. An MPI application executes in parallel, with each process running on its own Valgrind virtual machine. Each of these virtual machines implements an instance of the designed tracing tool. The tool instruments the original application and extracts the trace of the original (non-overlapped) execution, while at the same time, it generates what would be the trace of the potential (overlapped) execution. Then, Dimemas simulator uses the traces obtained from each MPI process and off-line reconstructs the application's timebehavior on a configurable parallel platform. Finally, Paraver visualizes the obtained time-behaviors, allowing to study the effects of the communication-computation overlap.

\section{A. Valgrind}

Valgrind [21] is a open-source dynamic binary translation framework suited to analyze memory usage. The system consists of a core, which presents a synthetic CPU in software on which all instructions are executed. Valgrind was originally designed to be a memory debugging tool, but today it is being used as a generic framework for designing dynamic analysis tools. In other words, Valgrind is a virtual machine that uses just-in-time (JIT) compilation techniques. The original code of an application never runs directly on the host processor. Instead, the code is first translated into a temporary, simpler, processor-neutral form called Intermediate Representation (IR). Then, a tool is free to do whatever transformations it wants on the IR, before Valgrind translates the IR back into machine code and lets the host processor run it.

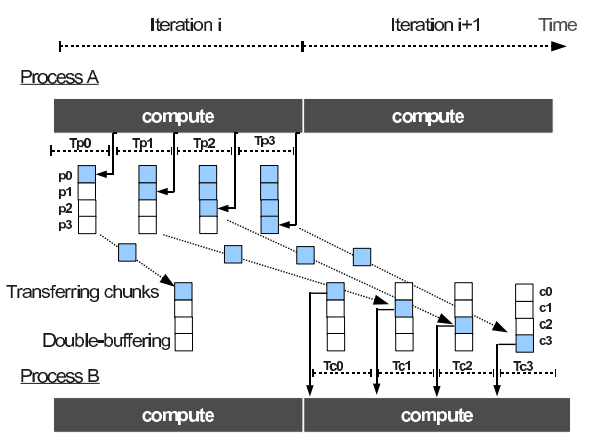

Figure 2. Case of overlapping communication and computation.

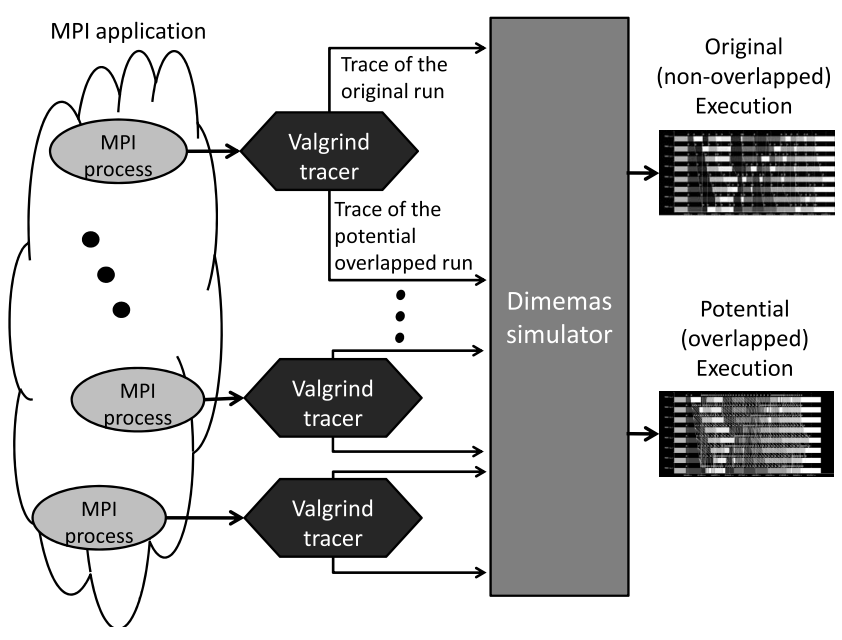

Figure 3. The simulation framework integrates Valgrind, Dimemas, and Paraver tools to easily analyze the communication-computation overlap in parallel applications.

\section{B. Dimemas}

Dimemas [13] is an open-source tracefile-based simulation tool for analysis of message-passing applications on a configurable parallel platform. It accurately predicts the performance of parallel applications in a particular system providing in this way support for their development and tuning. The communication model, validated in [14], consists of a linear model and some nonlinear effects, such as network congestion. The interconnect is parametrized by bandwidth, latency and the number of global buses (denoting how many messages are allowed to concurrently travel throughout the network). Also, each processor is characterized by the number of input/output ports that determine its injection rate to the network. 


\section{Implementation details}

Our major implementation effort consisted on designing a Valgrind tracing tool. The tracer leverages two key Valgrind functionalities for dynamic analysis of applications: wrapping function calls and tracking memory activities (loads and stores). The tool wraps each MPI call to read the parameters of the transfer and tracks each memory activity to monitor accesses to the transferred data. Furthermore, the tool needs additional data structures to keep track of the transfers' state and of production/consumption progress of every chunk. Finally, the tracer obtains time-stamps by scaling the number of executed instruction by the average MIPS rate observed in a real run.

In every run, the tracing tool generates one nonoverlapped (original) and two overlapped (potential) Dimemas traces. The non-overlapped trace describes the original execution of a legacy code by emitting two types of Dimemas trace records: computation records specifying the length of the original computation bursts in terms of the number of executed instructions; and communication records specifying the MPI message parameters. In addition to that tracing methodology, the first overlapped trace identifies within the original computation bursts, the points where partial data can be sent/is needed. Then it automatically splits each original message into various chunks and injects the chunked transmissions/receptions after the identified data is fully produced (for sends) and actually first needed (for receptions). Furthermore, in order to stress the influence of production/consumption patterns, the tool generates the second overlapped trace which assumes that the application's production/consumption patterns are ideal, as described in Section II. Thus, this tracing methodology models ideal computation pattern by uniformly distributing the chunked transmissions/receptions throughout the original computation bursts.

To model overlapped execution, the Valgrind tracer must intercept and process every application's load and store access. For every load in the application, the tool checks whether the requested element belongs to some incoming chunk that is not received so far. If so, the tracer emits a Dimemas wait-for-receive record for the corresponding chunk and marks that chunk as already received. Therefore, the tool guarantees that the wait for each incoming chunk is at the point where that chunk is needed for the first time. For every store in the application, the tool checks whether the accessed element belongs to some chunk to be sent. If so, it refreshes the record of the time of the chunk's last update, thereby maintaining the time of the last update for every chunk. The tool uses that information in the trace generation process described below.

Also, the tracer tool has to intercept each MPI call in order to reinterpret the original communication using new chunks. When the tracer intercepts a receive call, it emits a Dimemas non-blocking-receive record for each chunk of the original message. In this way, it initiates the transfers of chunks and proceeds, waiting for the chunks to be received as late as possible - when those chunks are actually needed for consumption. On intercepting a MPI send call, the tool consults the time of the last update of every chunk in the message, information generated during the production tracking. Using this data, the tracer emits a Dimemas send record of every chunk at the moment of the last update of that chunk, therefore generating the trace in which every chunk is sent at the exact moment when its final version is produced.

Note that collective communication operations are performed in Dimemas without assuming any collective hardware support on the network, so they are implemented as usual using multiple point-to-point MPI transfers.

\section{EXPERIMENTAL SETUP}

The application pool consists of Sweep3D [2], POP [1], Alya [16], SPECFEM3D [7], BT, and CG. Sweep3D is a wavefront application that solves a three-dimensional neutron transport problem. The problem size used is $50 \times 50 \times 50$ with $m k=10$. POP, Parallel Ocean Program, simulates oceans and their influence on climate. The input deck used is test with grid size of $192 \times 128 \times 20$. Alya is a multi-physics application that solves a variety of physics problems such as Convection-Diffusion-Reaction, Incompressible Flows, Compressible Flows, Turbulence, Bi-Phasic Flows and so on. We used the NASTIN module that solves the Incompressible Navier-Stokes. SPECFEM3D simulates earthquakes in complex three-dimensional geological models. The input deck used is test with 80 cells. And finally, BT and $\mathrm{CG}$ are two NAS parallel benchmarks. The problem used is class B.

The test-bed system consist of 64 PowerPC 9702.3 $\mathrm{GHz}$ processors interconnected with a Myrinet network that provides a unidirectional bandwidth of $250 \mathrm{MB} / \mathrm{s}$. This basic system configuration corresponds to the Marenostrum supercomputer that consists of 2,560 nodes achieving 63.8 Tflops on the Linpack benchmark. Table I shows the number of buses used in our experiments for each application. The number of buses has to be properly setup in the Dimemas simulator in order to match the simulated results with the real results of the application obtained from a real run on the Marenostrum supercomputer. In addition, the chunking technique in the overlapped case splits every MPI message in four chunks.

Table I

NUMBER OF NETWORK BUSES USED IN DIMEMAS FOR EACH APPLICATION.

$\frac{\text { Sweep3D }}{12} \frac{\text { POP }}{12} \frac{\text { Alya }}{11} \frac{\text { SPECFEM3D }}{8} \frac{\text { BT }}{22} \frac{\text { CG }}{6}$




\section{RESUlts}

Our framework provides a rich simulation environment to study the overlap. First, the tracer allows us to records the application's computation patterns, so we can further study their influence on the overlapping's potential. Second, Dimemas allows us to simulate various network configurations, so we can evaluate the impact of overlapping on future networks. Finally, Paraver allows to visualize the simulated time behaviors, so we qualitatively compare the non-overlapped and the overlapped execution.

For illustration purposes, Figure 4 shows the execution time for the first five iterations for the non-overlapped CG and the overlapped $\mathrm{CG}$ for four processes on the testbed system. As can be seen, the overlapped execution achieves $8 \%$ performance improvement with respect the nonoverlapped execution. With the Paraver tool we can easily investigate the cause of this improvement. As we can see, the performance improvement is mostly attributed to advancing the MPI transfer by sending the associated chunks earlier as we can see by the longer synchronization lines. This allows to partially overlap their communication delays with the computation of the succeeding chunks reducing significantly the Wait phases. This example shows how easy is to study the overlap in scientific applications.
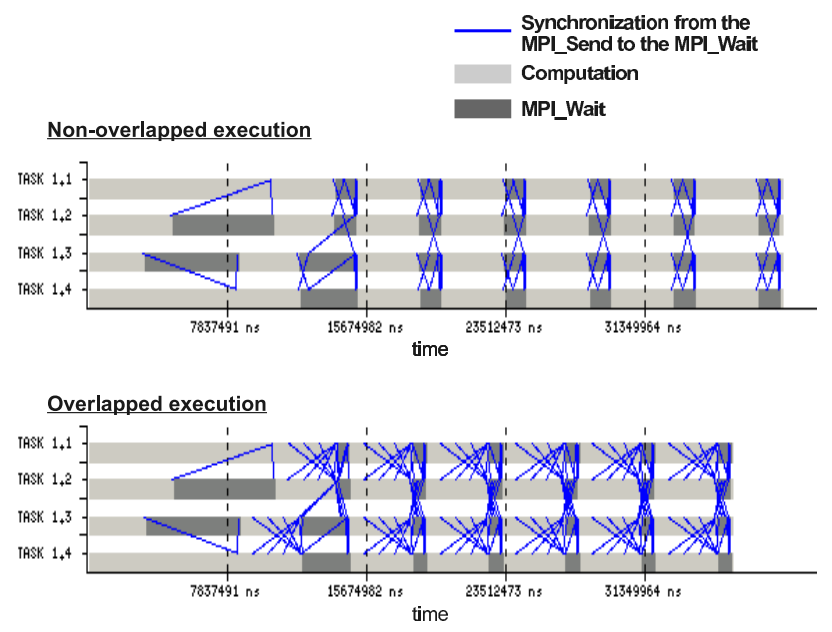

Figure 4. Paraver visualization for the non-overlapped and overlapped executions of NAS-CG.

\section{A. Production and consumption patterns}

The tracer also allows us to study how each process locally produces/consumes the MPI message. The tool identifies one production interval of a buffer as time between two consecutive sends of that buffer. Then, during the production interval, the tool intercepts all stores to the buffer and makes record of every store and the relative time within the interval when that store occurred. Similarly, the tracer defines one consumption interval as the period between two consecutive receives of the same buffer. During the consumption interval it collects records of every load and relative time within the consumption interval when that load occurred. These records can be plotted for analysis as shown in Figure 5 for Sweep3D, NAS-BT, and POP.

In particular, Figure 5(a) shows the production pattern for Sweep3D. The communicated buffer has 600 elements and all of them are revisited and accessed many times during one production interval. The first final version of any element is produced at $66.3 \%$ of the production interval, while the first quarter of the whole message is produced at $94.8 \%$ of the interval. In NAS-BT (Figure 5(b)), all the elements of the received buffer are loaded four times, each time in an extremely short interval, implying that the data is copied to some other location from where it is consumed during the computation. Patterns like these are extremely unfavorable for overlap. On the other hand, a property that may increase the potential of overlap is a presence of independent work [23], a portion of computation phase in which a program does not operate with the data involved in communication (Figure 5(c)).

Table II summarizes the production/consumption patterns for all applications analyzed in this paper. For each application it is shown the percentage of a computation phase where certain portions of a message are produced and consumed on average. These patterns show that: 1) the applications produce parts of the message late in the production interval (Table I(a)); 2) the applications consume parts of the message early in the consumption interval (Table I(b)). Therefore, the measured patterns provide little potential for advancing sends and post-postponing receptions.

\section{B. The benefits of overlap}

In this section we are going to quantify the benefits of overlapping. Specifically, we will first show that overlapping at the level of MPI always achieves speedup in legacy scientific applications. Second, we will demonstrate that overlap allows significant bandwidth relaxation, saying that the performance of the non-overlapped execution can be achieved with the overlapped execution on much lower bandwidth. And third, we will show that for some applications the performance improvement achieved by overlapping cannot be achieved by pure increasing the network bandwidth.

Overlap provides a small speedup for the real patterns and a decent speedup for the ideal patterns (Figure 6(a)). The unfavorable production/consumption patterns seriously limit the applications' overlapping potential. As anticipated from Table II, the real patterns allow speedup only in the case of NAS-CG. On the other hand, for modeled ideal patters, some applications achieve a significant speedup. The highest speedup is reached for Sweep3D due to the wavefront behavior of the application. For these type of ap- 


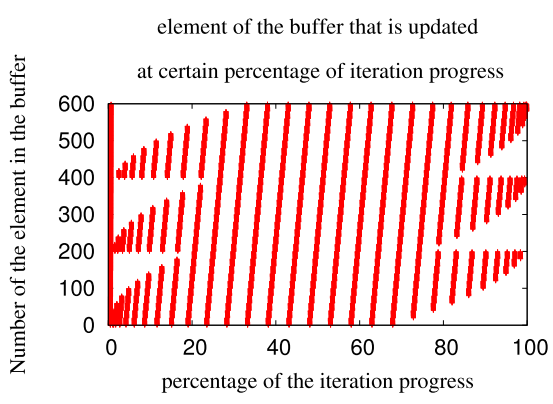

(a) SWEEP3D production pattern

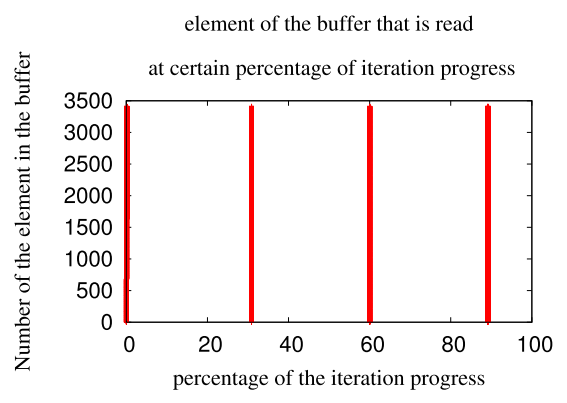

(b) NAS-BT consumption pattern

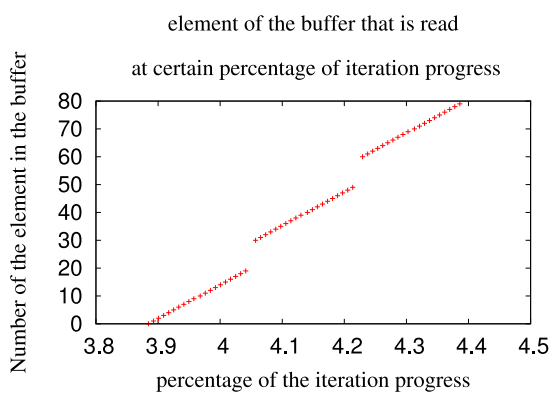

(c) POP consumption pattern

Figure interpretation: The $\mathrm{x}$ axis represents the normalized time within the corresponding computation interval (from start to end), while the $\mathrm{y}$ axis represents an element's offset within the transferred buffer. The points show when in computational interval each element was written for the production patterns or read for the consumption patterns.

Figure 5. Production and consumption patterns for Sweep3D, NAS-BT, and POP.

(a) Potential for advancing sends

\begin{tabular}{|l|l|l|l|l|}
\hline \hline & \multicolumn{4}{|c|}{$\begin{array}{c}\text { Percent of production phase } \\
\text { needed to produce } \\
\text { a part of a message }\end{array}$} \\
\hline \hline & $1^{\text {st }}$ element & quarter & half & whole \\
\hline ideal & $0 \%$ & $25 \%$ & $50 \%$ & $100 \%$ \\
\hline NAS-BT & $99.1 \%$ & $99.37 \%$ & $99.56 \%$ & $99.98 \%$ \\
\hline NAS-CG & $3.98 \%$ & $27.98 \%$ & $51.99 \%$ & $99.97 \%$ \\
\hline Sweep3D & $66.3 \%$ & $94.8 \%$ & $98.2 \%$ & $99.8 \%$ \\
\hline POP & $95.5 \%$ & $96.62 \%$ & $97.75 \%$ & $99.99 \%$ \\
\hline SPECFEM3D & $95.3 \%$ & $96.48 \%$ & $97.65 \%$ & $98.87 \%$ \\
\hline Alya & & & & $98.8 \%$ \\
\hline \hline
\end{tabular}

(b) Potential for Post-postponing receptions

\begin{tabular}{|l|l|l|l|}
\hline \hline & \multicolumn{3}{|c|}{$\begin{array}{c}\text { Percent of consumption phase } \\
\text { that can be passed } \\
\text { upon reception of a part of a message }\end{array}$} \\
\hline \hline & nothing & quarter & half \\
\hline ideal & $0 \%$ & $25 \%$ & $50 \%$ \\
\hline NAS-BT & $13.68 \%$ & $13.71 \%$ & $13.74 \%$ \\
\hline NAS-CG & $2.175 \%$ & $18.35 \%$ & $34.53 \%$ \\
\hline Sweep3D & $0.02 \%$ & $0.003 \%$ & $0.004 \%$ \\
\hline POP & $3.525 \%$ & $3.53 \%$ & $3.534 \%$ \\
\hline SPECFEM & $0.032 \%$ & $0.034 \%$ & $0.036 \%$ \\
\hline Alya & $0.4 \%$ & & \\
\hline \hline
\end{tabular}

Note: Since the instrumented kernel of Alya communicate mainly using MPI reduction collectives of length of one element, these transfers cannot be chunked into partial ones, so the tables show no results for a part of a message.

Table II
PRoduction AND CONSUMPTION AVERAGE PATtERns.

plications (concurrent, pipeline), the chunking mechanisms of overlap causes finer-grain dependencies among processes and potentially increases parallelism of different processes.

The biggest benefit of overlap is that it allows to significantly relax network bandwidth without consequently degrading the performance (Figure 6(b)). Figure 6(b) shows that in order to achieve the performance of the nonoverlapped execution on $250 \mathrm{MB} / \mathrm{s}$, the overlapped execution needs much less bandwidth. Again, Sweep3D benefits from overlap the most and allows to reduce the network bandwidth to $11.75 \mathrm{MB} / \mathrm{s}$ and maintain the performance of the original execution. Relaxation of network bandwidth is very important because it means that in order to achieve the performance of the original execution on a state-of-the-art network, the overlapped execution requires a much cheaper network.

Finally, the benefits achieved by applying automatic overlap sometimes cannot be reached by simply increasing the network bandwidth. Figure 6(c) shows the bandwidth required by the non-overlapped execution in order to achieve the performance of the overlapped execution at $250 \mathrm{MB} / \mathrm{s}$. In other words it demonstrates what is the overlap's equivalent in increased network bandwidth. The result of Sweep3D shows that for some applications the performance of the overlapped execution cannot be achieved with non-overlapped execution on any bandwidth. Also, it is interesting to note that overlap brings little speedup in SPECFEM3D (Figure 6(a)), but the benefits achieved by overlap are equivalent to benefits that could be achieved by increasing the network bandwidth almost four times.

\section{RELATED WORK}

Previous research in the field of overlapping communication and computation could roughly be categorized in three directions. These are: 1) exploring state-of-the-art support for exploiting overlap; 2) exploring overlapping techniques; and 3) measuring the potential for overlap that is present in applications.

First, several studies evaluated the overlapping capability of different processors, networks and programming lan- 


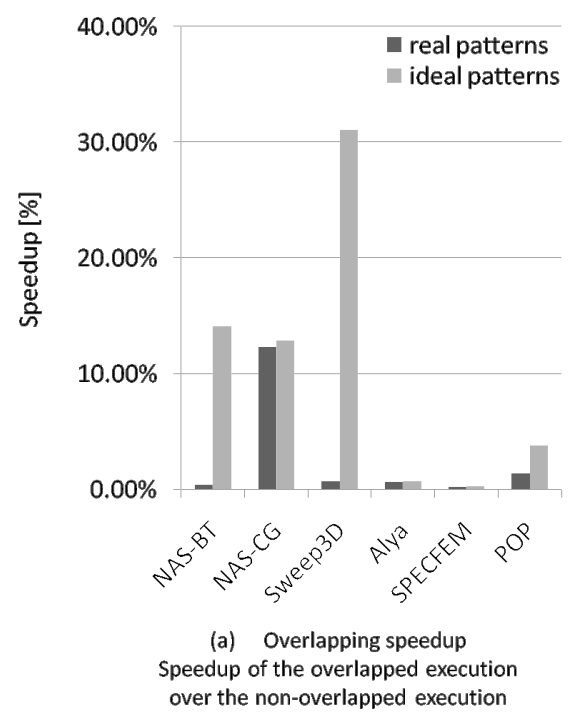

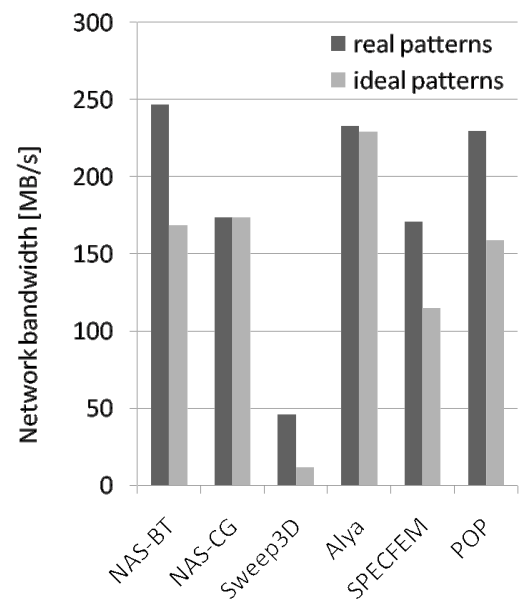

(b) Relaxation of network bandwidth: Network bandwidth at which the overlapped execution gives the same performance as the non-overlapped at $250 \mathrm{MB} / \mathrm{s}$

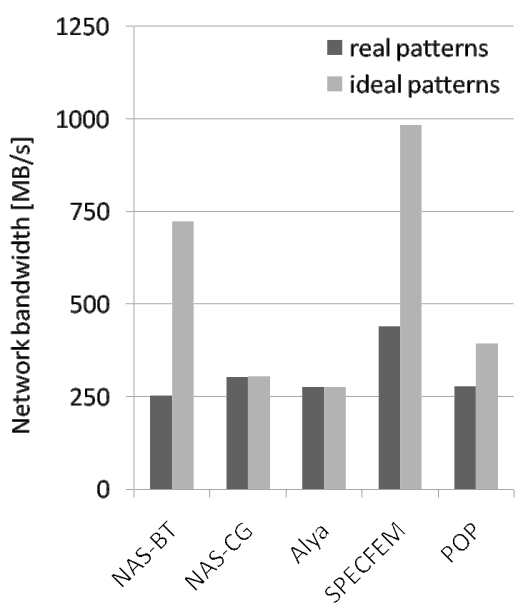

(c) Equivalent bandwidth advancement: Network bandwidth at which the non-overlapped execution gives the same performance as the overlapped at $250 \mathrm{MB} / \mathrm{s}$

Note: In Figure (c), the equivalent bandwidth advancement for Sweep3D for both real and ideal patterns tends to infinity, and therefore it is not shown.

Figure 6. Simulation of the overlapped executions on the real and ideal production/consumption patterns.

guages. Sohn et al. [24] tested various multiprocessors and compared their overlapping efficiency. Furthermore, Brightwell et al. [5] quantified in detail how performance is influenced by the network properties of overlap, offload and independent progress. Later work studied many MPI implementations and showed that their overlapping abilities are different [18], [6]. Further research led to design of PGAS languages[8], [9], new programming languages that decouple communication and synchronization to achieve higher overlap. Afterward, Bell at al. [3] showed overlapping advantages of light one-sided transfers implemented in UPC. In this work, we assume that the used underlying communication layer is fully capable of overlapping communication and computation.

Second, many research efforts explored implementation issues of overlapping techniques. In an effort to hide communication delays, Leu et al. [19] identified overlapping as a technique that can provide maximum application speedup of two. Later, Danalis et al. [11] defined general code restructuring approaches that lead to better overlap in applications that exhibit limited dependencies among iterations. Hoefler et al. [15] proved overlapping potential of nonblocking collective communications in MPI. Furthermore, Das et al. [12] extended a compiler so it can postpones receptions (sinks waits) in MPI applications, while Iancu et al. [17] changed UPC runtime library to implement demand- driven synchronization, automatic message strip mining and message scheduling. However, mentioned efforts fail to clearly determine the potential benefits of their overlapping techniques because they fail to isolate the overlapping effect from the implementations' side-effects such as: changed locality (cache and TLB misses), non-deterministic events (OS daemons, preemptions, interferences in a shared resource), etc. On the other hand, our simulation can measure isolated impact of overlap, since the simulation framework introduces overlapping mechanisms without impacting other execution properties.

Third, there was little effort to identify the potential for overlap in applications. Sancho et al. [23] provided a theoretical estimation of the overlapping potential in scientific codes by modeling a real application with one iterative loop and parameters that roughly describe the computation pattern. Our paper continues Sancho's work by designing a simulation frameworks that brings the following advances in the study of the overlapping potential: 1) automatic simulation without the need to understand the studied application; 2) simulation for diverse network configurations; and 3) visualization of the simulated time behaviors.

\section{CONCLUSIONS AND FUTURE WORK}

This study is the first simulation-based approach to quantify the potential benefits of overlap. We designed a simu- 
lation framework that can simulate applications' overlapped execution automatically without the need to know or change the applications legacy code. Compared to the previous studies in the field, our framework accounts for more delicate application properties and allows to study overlap on diverse network configurations. Finally, the framework provides useful visualization of the simulated time behaviors, so we can qualitatively compare the non-overlapped and the overlapped execution.

Overlap brings significant execution advancements, especially in the case of favorable production/consumption patterns. We showed that real scientific applications have diverse computation patterns that are often unfavorable for overlap. We confirm that for favorable patterns, overlap achieves two benefits: execution speedup; and relaxation of bandwidth without consequently degradation of performance. Moreover, in some applications the benefits achieved by overlap cannot be reached by simple increasing network bandwidth.

Our study can be useful for researchers in the field to understand better the potential and the mechanism of overlap. The simulation framework can be useful to researchers that try to actually overlap communication and computation since it allows them to estimate the impact of their proposed implementations. Furthermore, they can use the framework as development support because Paraver visualization could help them identify specific bottlenecks in their implementations.

We plan to continue developing our simulation framework in order to explore other overlapping techniques. The results of this paper showed us that overlap at the level of MPI calls is very limited by application's production/consumption patterns. Therefore, at first place, we want to find ways to exploit overlap at the level of the application's computation phases.

\section{REFERENCES}

[1] POP: Parallel Ocean Program. http://climate.lanl.gov/Models/POP/, 2008.

[2] SWEEP3D: 3D Discrete Ordinates Neutron Transport . www.c3.lanl.gov/pal/software/sweep3d/sweep3d, 2008.

[3] C. Bell, D. Bonachea, R. Nishtala, and Yelick. K. Optimizing bandwidth limited problems using one-sided communication and overlap. In IPDPS. 2006.

[4] N. J. Boden, D. Cohen, R. E. F. A. E. Kulawik, J. N. Seitz, C. L. ans Seizovic, and W-K Su. Myrinet: a gigabit-per-second local area network. IEEE Micro, 15(1):29-36, 1995.

[5] R. Brightwell, R. Riesen, and K. D. Underwood. Analyzing the Impact of Overlap, Offload, and Independent Progress for Message Passing Interface Applications. The International Journal on High Performance Computing Applications, 19(2):103-117, 2005.
[6] R. Brightwell and K. D. Underwood. An initial analysis of the impact of overlap and independent progress for MPI. In 9th European PVM/MPI Users' Group Meeting. 2004.

[7] L. Carrington, D. Komatitsch, M. Laurenzano, M. Tikir, D. Michea, N. Le Goff, A. Snavely, and J. Tromp. Highfrequency simulations of global seismic wave propagation using SPECFEM3D_GLOBE. In Supercomputing, SC 08. 2008.

[8] W. Chen, C. Iancu, and K. Yelick. Communication optimizations for fine-grained UPC applications. In PACT. 2005.

[9] C. Coarfa, Y. Dotsenko, J. Eckhardt, and J. Mellor-Crummey. Co-Array Fortran performance and potential: An NPB experimental study. In International Workshop on Lenguages and Compilers for Parallel Computing. 2003.

[10] W Dally. Principles and Practices of Interconnection Networks. Morgan Kaufmann Publishers, 2004.

[11] A. Danalis, K-Y Kim, L. Pollock, and M. Swany. Transformations to Parallel Codes for Communication-Computation Overlap. In Supercomputing, SC 05. 2005.

[12] D. Das, M. Gupta, R. Ravindran, W. Shivani, P. Sivakeshava, and R. Uppal. Compiler-controlled extraction of computationcommunication overlap in MPI applications. In IPDPS, pages 1-8, 2008.

[13] Dimemas. Dimemas: Performance Analysis Tool for Parallel Programs and Platforms. http://www.bsc.es/plantillaA.php?cat_id=475/, 2008.

[14] S. Girona, J. Labarta, and R.M. Badia. Validation of Dimemas communication model for MPI collective operations. In EuroPVM/MPI'2000. 2000.

[15] T. Hoefler, A. Lumsdaine, and W. Rehm. Implementation and performance analysis of non-blocking collective operations for MPI. In SC, page 52, 2007.

[16] G. Houzeaux, B. Eguzkitza, and M. Vazquez. A variational multiscale model for the advection-diffusion-reaction equation. Communications in Numerical Methods in Engineering, 2007.

[17] C. Iancu, P. Husbands, and P. Hargrowe. HUNTing the overlap. In PACT. 2005.

[18] W. Lawry, C. Wilson, A. B. Maccabe, and R. Brightwell. COMB: A portable benchmark suite for assessing MPI overlap. In Cluster Computing. 2002.

[19] J. Leu, D. P. Agrawal, and J. Mauney. Modeling of parallel software for efficient computation-communication overlap. In Fall Joiny Computer Conference. IEEE Press, 1987.

[20] mellanox. Mellanox InfiniBand IV Switch Silicon. http://www.mellanox.com, 2010.

[21] N. Nethercote and J. Seward. Valgrind. http://valgrind.org/, 2008. 
[22] V. Pillet, J. Labarta, T. Cortes, and S. Girona. PARAVER: A Tool to Visualize and Analyze Parallel Code. In WoTUG-18. 1995.

[23] J.C. Sancho, K.J. Barker, D.J. Kerbyson, and K. Davis. Quantifying the Potential Benefit of Overlapping Communication and Computation in Large-Scale Scientific Applications. In Supercomputing, SC 06. 2006.

[24] A. Sohn, J. Ku, Y. Kodama, M. Sato, H. Sakane, H Yamana, S. Sakai, and Y. Yamaguhi. Identifying the capability of overlapping computation and communication. In PACT. 1996. 